\title{
Un exemple de résolution analogique, à l'aide d'un réseau R.C., d'un problème de diffusivité dans une nappe aquifère
}

\author{
An example of the solution \\ of an aquifer diffusivity problem \\ by analogy with the aid of an 'R.C. network'
}

\author{
PAR A. LAGARDE, \\ INSTITUT FRANGAIS DU PÉTROLE
}

\begin{abstract}
Après avoir brièvement indiqué quelques généralités sur l'analogie électrique mise en cause, on décrit les caractéristiques particulières du réseau R.C. utilisé à l'Institut Français du Pétrole, réseau qu'on appelle communément «analyseur électrique» ou «simulateur de gisements $\gg$.

On expose ensuite le problème d'une injection et d'un soutirage alternés dans une nappe aquifère, nappe dont on suppose connues les caractéristiques de transmissivité et d'emmagasinement. On montre alors comment ce problème est résolu analogiquement malgré la présence de parties libres dans la nappe et malgré l'impossibilité de pouvoir représenter correctement l'ensemble du bassin d'alimentation.

On étudie alors rapidement les différentes réponses fournies par l'appareil aux différentes hypothèses envisagées concernant les débits, ce qui permet de sélectionner parmi le lot testé celle répondant le mieux aux exigences requises. On donne enfin une idée des problèmes de diffusivité similaires qui peuvent être traités par le simulateur.
\end{abstract}

\section{I. - GÉNERALITÉS}

\section{Principe.}

Le réseau R. C. de l'Institut Français du Pétrole est un calculateur analogique direct, ou

\begin{abstract}
A few general remarks on the electrical analogy considered are followed by a description of the special characteristics of the "R. $C$. network" used at the "Institut. Français du P'étrole", which is also commonly referred to as an "electrical analyser" or "deposit simulator". The problem of alternating artificial recharging and discharging of an aquifer of assumed known transmissivity and storage characteristies in then described, and it is shorvn how a solution can be oblained by analogy, even though the water lable also inchudes unconfined surfaces and despite the impossibility of adequately simulating the whole of the basin supplying the inflows.

The responses of the apparalus to the varions discharge assumption made are then bricfly studied and the one most suilable for the considered requirements selected. Finally, general indications are given of other similar diffusivily problems for which the apparalus can be used.
\end{abstract}

simulateur, spécialement concu pour l'étude des gisements d'huile sous-saturée en gaz, e'est-àdire dans lesquels la compressibilité de l'huile est supposée ne pas varier dans la gamme des pressions considérées. Comme dans tout simulateur, il y a correspondance entre chaque élément,

(*) Ce réseau R.C. a été construit dans le cadre du programme de l'Association de Recherche sur les Techniques de Forage et de Production (A.R.T.F.P.). 
ou chaque grandeur, du calculateur analogique et du système original. Dans notre cas cette correspondance r'ésulte de l'analogie entre les lois de l'écoulement des fluides dans un milieu poreux et celles de l'écoulement du courant électrique dans les réseaux passifs, que l'on peut écrire ainsi :

\section{a) Ecoulement des fluides :}

$$
\text { loi de Darcy : } \overrightarrow{\mathrm{V}}=-\frac{k}{\varphi^{\prime}}-\overline{\operatorname{grad} \mathrm{P}}
$$

équation de continuité : div $\overrightarrow{\varphi \varphi \vec{V}}=-\frac{\partial(\varphi \rho)}{\partial t}$

b) Ecoulement du courant telectrigue :

$$
\text { loi d'Ohm: } \quad \vec{j}=-\sigma \overrightarrow{\operatorname{grad} E}
$$

loi de Maxwell $: \operatorname{div} \vec{j}=-\mathrm{C} \frac{\partial \mathrm{E}}{\partial t}$

(équation de continuité)

où l'on a respectivement :

$\mathrm{V}$ vitesse moyenne du fluide dans le milieu poreux ;

k perméabilité du milieu poreux au fluide considéré;

$\varphi$ porosité du milieu poreux;

$\mu$ riscosité du fluide considéré saturant le milieu poreux;

P pression;

$\rho$ masse spécifique du fluide considéré;

j vecteur densité de courant;

$\sigma$ conductivité électrique;

E tension électrique;

C capacité électrique.

En introduisant l'équation d'état du fluide supposé subir une évolution isotherme :

$$
f(\mathrm{P}, \mathrm{\rho})=0
$$

on peut éliminer la masse spécifique de l'équation de continuité du fluide. Cette élimination est aisée si l'on suppose que le fluide est peu compressible (compressibilité très faible et dépendant peu de la pression) et que les variations de pression autour de la pression moyenne sont faibles, ce qui permet d'écrire l'équation d'état sous la forme :

$$
\rho \varphi=\rho_{0} \varphi_{0} e^{-\beta_{t}\left(p-p_{0}\right)} \# \rho_{0} \varphi_{0}\left[1+\beta_{t}\left(p_{0}-p\right)\right]
$$

et par suite l'équation de continuité du fluide sous la forme :

$$
\operatorname{div} \overrightarrow{\varphi v}=-\cdots \varphi \beta_{t} \frac{\partial \mathrm{P}}{\partial t}
$$

On voit ainsi apparaître l'analogie entre la capacité spécifique d'expansion du fluide et la capacité électrique $C$.

On remarque que si on suppose la compressibilité du fluide rigoureusement nulle, on retrouve alors l'analogie entre les écoulements permanents des fluides en milieu poreux et ceux du courant électrique dans les conducteurs simplement résistants (cuve électrolytique, réseau de résistances, papier conducteur).

Dans le cas, qui nous intéresse, des mouvements transitoires, on voit qu'il y a analogie entre pression et tension, résistivité électrique $1 / \sigma$ et résistivité hydraulique $\mu / k$, capacité d'expansion $\varphi \beta$ et capacité électrique $\mathrm{C}$, analogies quicontiennent implicitement celles entre débit des fluides $Q$ et intensité électrique $i$, et entre temps réel et temps électrique.

A partir de l'analogie que nous venons de présenter, la simulation de l'écoulement des fluides dans les milieux poreux se fait en discrétisant les variables d'espace $x$ et $y$ par passage aux différences finies des équations considérées. Ainsi, si on considère un bloc de milieu poreux de surface latérale $A$, de longueur $L$, de perméabilité $k$, traversé longitudinalement par un fluide de viscosité $\mu$, ce bloc sera simulé électriquement par une résistance $R$ et une capacité $C$ dont les valeurs seront respectivement proportionnelles aux grandeurs physiques analogues après discrétisation, soit :

$$
\mathrm{R}_{f}=(\mu \mathrm{L} / \mathrm{Ak}) \text { et } \mathrm{C}_{f}=\mathrm{AL} \varphi \beta
$$

Si l'on se fixe les coefficients d'analogie entre ces grandeurs et les grandeurs électriques, l'échelle de représentation des temps sera fixée, puisque le produit $R C$, ou la quantité $\mu L^{2} \varphi \beta / k$, sont homogènes à un temps. Il ne reste plus alors, pour définir complètement l'analogie, qu'à fixer la correspondance entre l'un des couples $Q-i$ et $\mathrm{P}$-E, l'autre en découlant automatiquement.

Il faut remarquer que l'analogie R. C. n'est qu'un cas particulier de l'analogie des réseaux R. C. L. qui permet de résoudre différents types d'équations aux dérivées partielles, et en particulier les équations de Maxwell. 


\section{Application aux nappes captives et libres.}

a) Nappes captuves: la simulation réalisée sur le réseau R.C. de l'Institut Francais du Pétrole est une simulation bidimensionnelle; on s'intéresse aux couches minces, pour lesquelles les équipotentielles sont pratiquement des cylindres à génératrices verticales; quelquefois, pour des cas théoriques, les deux dimensions peuvent être $Z$ et $r$, en supposant une symétrie de révolution; mais la grande majorité des applications est une simulation de mouvements supposés plans dans des systèmes supposés peu épais, ce qui est le cas de beaucoup de gisements d'huile.

En faisant apparaitre la hauteur de la couche, l'analogie des réseaux R. C. permet de résoudre ainsi l'équation :

$$
\operatorname{div}\left(\frac{h k}{\mu} \overrightarrow{\operatorname{grad} \overrightarrow{\mathbf{P}}}\right)=h \beta \varphi \frac{\partial \mathrm{P}}{\partial t}
$$

combinaison de l'équation de Darcy et de l'équation de continuité.

Lorsque le milieu poreux est homogène et que la hauteur est constante, cette équation devient l'équation bien connue :

$$
\Delta \mathrm{P}=\frac{1}{k} \frac{\partial \mathrm{P}}{\partial t} \text { où } \mathrm{K}=\frac{k}{\varphi \mu \beta},
$$

diffusivité hydraulique, n'est autre que le rapport de la transmissivité hydraulique :

$$
\mathrm{T}=(h k \rho g / \mu)
$$

et $d u$ coefficient d'emmagasinement $S=h \beta \varphi p g$.

Plus que l'équation de diffusivité proprement dite, le simulateur de gisements résout l'équation (1) : il tient compte ainsi des variations géographiques des caractéristiques physiques $d u$ milieu poreux, hauteur $h$, perméabilité $k$, porosité $\varphi$, mais il suppose que la compressibilité $\beta$ et la viscosité $\mu$ du fluide sont constantes dans le temps.

Nous avons parlé de gisements minces et de mouvements plans; si le pendage des couches est malgré lout important, il est fait abstraction des phénomènes de gravité; il suffit de parler non plus de pressions, mais de potentiels; de toute façon, le simulateur fournit des solutions rapportées à un état initial en terme de variations; ainsi, dans Ie cas de couches parfaitemennt planes, l'analogie peut ne pas respecter la pression initiale du fluide : étant donnée la linéarité en $\mathrm{P}$ de l'équation de diffusion, il suffit de connaître les variations de pression à partir de l'état d'équilibre pris comme référence. b) NAPpes Libres : dans le cas des nappes libres, l'hypothèse de Dupuit permet d'écrire une équation identique à l'expression de la loi de Darcy :

$$
\overrightarrow{\mathrm{V}}=-\frac{k}{\mu} \overrightarrow{\operatorname{grad~H}}
$$

où $\mathrm{H}$ est alors la cote de la surface libre par rapport à un plan de référence.

D'autre part, l'équation de continuité s'écrit pour les nappes libres:

$$
\operatorname{div} \overrightarrow{h V}=-\varphi^{\prime} \frac{\partial h}{\partial t}
$$

où $h$ est la hauteur d'eau au-dessus du mur imperméable et $\varphi^{\prime}$ la porosité de la partie libre.

Si on suppose que $d \mathbf{H}=d h$ est petit devant $h$ (opération de linéarisation), la combinaison de ces deux équations donne l'équation de diffusivité pour les nappes libres :

$$
\operatorname{div}\left(\frac{h k}{\mu} \overrightarrow{\operatorname{grad~H}}\right)=\varphi^{\prime} \frac{\partial \mathrm{H}}{\partial t}
$$

équation identique à celle établie pour les gisements ou nappes captives (équation (1)), à ceci près que, cette fois, la capacité d'expansion $h \varphi p \beta$ est remplacée par la porosité efficace $\varphi^{\prime}$. Analogiquement, cela signifie que les parties libres seront représentées sur le simulateur par des capacités électriques dans le rapport $\left(\varphi^{\prime} / h \varphi \beta\right)$ avec celles des parties captives, e'est-à-dire par des capacités considérablement plus importantes.

Enfin, l'analogie entre charges et tensions électriques devient une analogie entre variations de niveau et ces mêmes tensions électriques.

\section{Description des caractéristiques du simu- lateur.}

a) FrÉQuence : le simulateur de gisements est du type répétitif ou à faible constante de temps. Sa fréquence de fonctionnement est de $250 \mathrm{~Hz}$, soit une période de 4 millisecondes; en fait, la période utile à la simulation est de 2 millisecondes, les deux autres millisecondes étant utilisées pour la recharge des circuits. L'avantage de l'utilisation d'une fréquence élevée de fonctionnement est d'obtenir une réponse instantanée à toute modification dans le réseau, par simple visualisation sur oscilloscope; ce système constitue en quelque sorte un moyen détourné de stocker les résultats, puisque, au contraire des simulateurs à grande constante de temps, où il est nécessaire de «sortir» tous les renseigne- 
ments nécessaires au moment de l'expérience, faute d'avoir à la recommencer, à tout instant le réseau est prêt à délirrer l'information dont on est avide. Un autre avantage de cette fréquence élevée de fonctionnement est de diminuer l'importance des capacités, donc d'éliminer pratiquement tout risque de fuites; parallèlement à cet avantage, il faut remarquer qu'on ne peut descendre à des valeurs trop faibles des capacités sans risquer de voir celles-ci devenir inférieures aux capacités parasites qu'on ne peut éviter.

b) Réseau R. C. : le réseau R. C. proprement dit est constitué de 158 cellules ou mailles contenant chacune un jeu de capacités et deux jeux de résistances. Parmi ces 158 mailles, 128 sont «télécommandées » à partir du pupitre central où se trouvent oscilloscope et enregistreur : l'affichage des valeurs désirées pour les résistanees $\mathrm{R}_{x} \mathrm{R}_{y}$ et la capacité de la maille se fait ainsi à distance, les mailles en question étant équipees de relais à mémoire magnétique servant à la sélection des éléments suivant le principe de la boite de poids. Dans ces mailles, les gammes de variation des éléments sont respectivement de $1000 \mathrm{pF}$ à $100000 \mathrm{pF}$ pour les capacités, compte tenu des capacités parasites, et de 10 à $100000 \Omega$ pour les résistances. La valeur des capacités de chaque maille peut être contròlée à l'aide d'on capacimètre de précision.

Les 30 mailles restantes sont à sélection manuelle et présentent des gammes de variation des éléments respectivement de $100 \mathrm{pF}$ à $10000 \mathrm{pF}$ de 10 en $10 \mathrm{pF}$ pour les capacités ( $\mathrm{y}$ compris les parasites) et de 100 à $100000 \Omega$ pour les résistances.

c) Conditions aux puits: la simulation de l'histoire passée et des prévisions de vie du gisement se fait à partir de fonctions production et injection, au nombre de 8, qui, à l'emplacement correspondant aux puits du gisement sur le réseau R. C., soutirent ou injectent le courant électrique suivant ce que l'on désire simuler.

En fait, bien que, dans l'analogie considérée, le temps soit une variable absolument continue quand on désire résoudre un problème à débit constant, les fonctions production et injection ont été concues pour pouvoir représenter une loi de débit en escalier pouvant comporter jusqu'à 48 paliers ou steps de temps, paliers aussi bien réglables en durée qu'en amplitude (plages de 20 à $120 \mu \mathrm{s}$ en durée, de $0,05 \mathrm{~mA}$ à $2,5 \mathrm{~mA}$ en amplitude). Ainsi, l'analyseur électrique, capable de fournir la fonction d'influence de chacun des puits pris isolément dans le gisement (c'est-àdire la loi de pression, au puits, correspondant à un débit unité) est aussi capable de combiner les fonctions d'influence de plusieurs puits selon l'histoire des débits de ces puits, par application instantanée du théorème de superposition. Il fait élégamment le travail des ordinateurs à qui on donne souvent à digérer et combiner les fonctions d'influence fournies par un calculateur analogique à puits unique.

La réalisation de ces fonctions production et injection est assez simple pour le manipulateur; une fois l'échelle des temps choisie, grâce à l'emploi combiné 'd'un oscilloscope à grande bande passante et d'un système de synchronisation extérieur, il est possible de régler un par un chaque step de temps en largeur et en amplitude. Electroniquement, la réalisation et la juxtaposition des différents paliers sont réalisées assez facilement : en effet, le premier palier est engendré par une impulsion synchronisée qui est applicquée à un circuit de retard (bascule monostable); celui-ci permet de réaliser un palier de durée et d'amplitude variables, réglées par le manipulateur; à la fin de ce palier, une nouvelle impulsion engendre à travers un second circuit intégrateur, identique au précédent, un second palier réglable. La première impulsion est donnée par le générateur d'impulsions au début de chaque cycle de 4 millisecondes. La seconde impulsion est obtenue par un système différenciateur et inverseur agissant sur le premier palier. Enfin, les paliers sont juxtaposés à l'aide de cathode-follower, de telle sorte qu'ils n'ont aucune réaction les uns sur les autres quand on les règle un à un.

d) Conditrons aUd Limites: dans les types de problème que nous sommes amenés à traiter, les conditions aux limites sont de deux types extrêmement classiques :

$1^{\circ}$ les limites imperméables $[(\partial \varphi / \partial n)=0]$, sont réalisées par arrêt de maillage et les failles étanches $(h=0)$ par déconnection des résistances reliant les mailles;

$2^{\circ}$ les contours d'alimentation $[P(t)=$ Cte $]$ sont réalisés par un système permettant de maintenir une tension constante aux points désirés.

e) Systèmes de Mesure : les systèmes de mesure sont essentiellement constitués par des oscilloscopes. Grâce à une caméra polarö̈d, il est possible d'obtenir pratiquement instantanément les photos témoins de l'évolution des pressions aux points désirés. Si une plus grande précision (précision absolue) est demandée, le simulateur est équipé d'un enregistreur SEFrAM à double voie, qui, fonctionnant par échantillonnage, donne les courhes de pression avec une meilleure définition et une précision de $1 \%$ environ. 


\section{II. - APPLICATION A L'ÉTUDE DE LA NAPPE AQUIFËE DE LA VALLEE DE LA HAUTE-DEULE ${ }^{*}$ )}

\section{Exposé du problème.}

Le problème dont nous allons exposer les données concerne la nappe aquifère de la craie de la vallée de la Hautc-Deule, nappe essentielle à l'alimentation en eau du département du Nord, puisque, en dehors des forages industriels, elle alimente les prélèvements de la Société des Eaux du Nord et de la ville de Lille pour desservir l'agglomération lilloise. En 1962, elle a fourni $230000 \mathrm{~m}^{3} / \mathrm{jour}$, mais les autorisations accordées doivent porter ce débit à $270000 \mathrm{~m}^{3} /$ jour en 1963, alors que les évaluations concernant les possibilités de la nappe à partir de la surface du bassin versant et des données hydrologiques sont de $280000 \mathrm{~m}^{3} /$ jour (à $10 \%$ près). Compte tenu de ce que, les hesoins se développant, de nouveaux besoins sont encore à prévoir, la nappe elle-même ne pourra suffire. Comme des adductions d'eau à partir de nouveaux gîtes aquifères sont très onéreuses, les gîtes déjà reconnus étant très éloignés, une solution au problème est la réalimentation artificielle de la nappe pour compenser l'augmentation du débit qui doit avoir lieu dans la région des Ansereuilles; une telle réalimentation est en effet possible par réinjection de l'eau du canal de la Deule au voisinage de celui-ci. Le but de l'étude que nous présentons était essentiellement de répondre aux questions que posent cette réinjection, à savoir la recherche des meilleurs emplacements des puits d'injection et de la meilleure ventilation des débits totaux d'injection et de soutirage dans le temps, pour que la baisse de niveau dans la nappe ne compromette pas localement les possibilités de pompage. A ce stade du prohlème, et pour mieux le comprendre, il nous semble utile de donner une description du gîte aquifère. Celui-ci a été étudié par le professeur G. Waterlot, à qui nous empruntons l'essentiel de ce qui suit.

\section{Description du gîte aquifère.}

Ce gîte est un véritable piège à eau grâce à la présence d'un synclinal formant une vaste cuvette; l'eau peut s'y accumuler d'autant mieux

( $\left.{ }^{\star}\right)$ L'arrondissement minéralogique de Douai a bien voulu nous autoriser à diffuser les éléments de cette application.

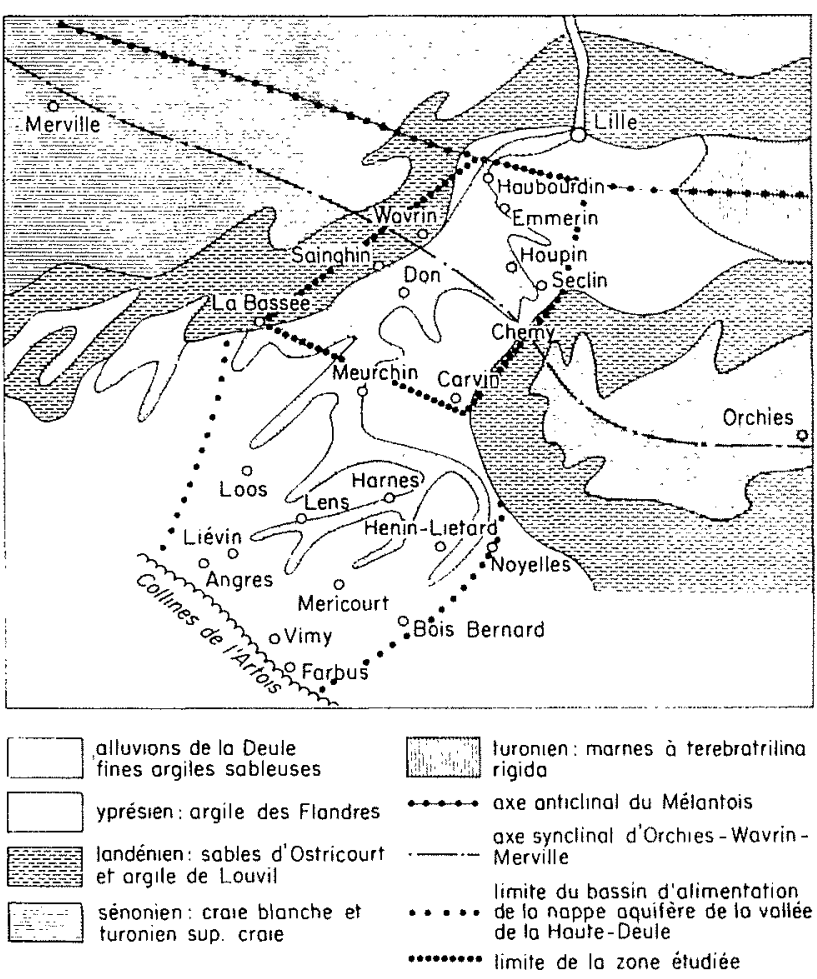

FIG. 1

Vallée de la Haute-Deule (d'après G. Waterlot).

qu'elle y est retenue au nord-est par l'anticlinal du Mélantois formant barrage souterrain et qu'au sud-ouest la vallée qui sert de bassin d'alimentalion s'étend sur une grande distance. Ce seuil du Mélantois fait office de déversoir de l'excédent de la nappe dans la banlieue lilloise et, par suite, le niveau de l'eau y est extrêmement important. Cette eau est contenue dans la

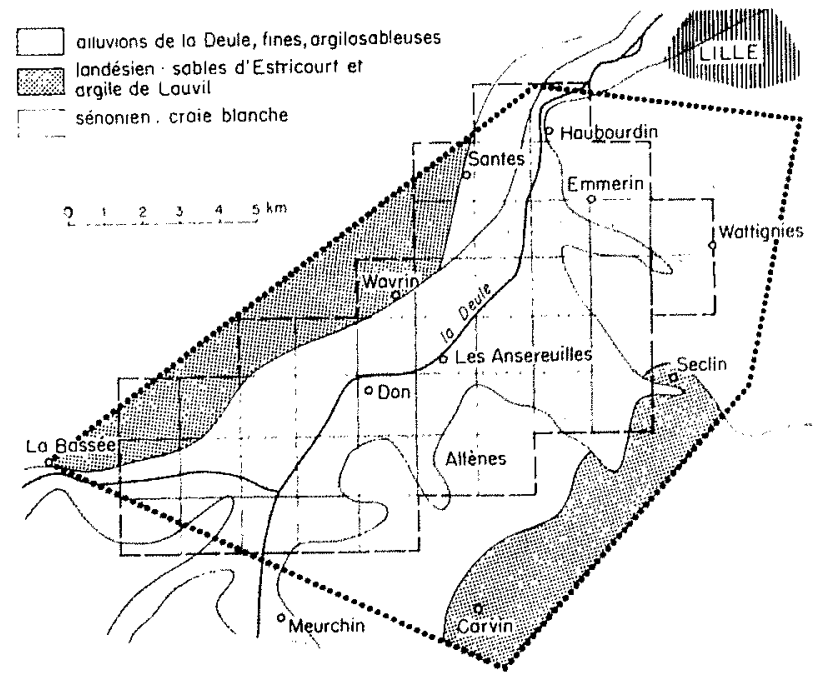

Fig. 2 
craie du sénonien et du turonien supérieur. Dans une partie de la région, la craie affleure et la nappe est ainsi libre; ailleurs, elle est recouverte par des alluvions quaternaires argilosableuses pratiquement imperméables et la nappe peut ainsi être considérée comme captive; au nord-ouest et an sud-est, la formation aquifère s'enfonce sous des terrains tertiaires imperméables; au fur et à mesure que l'on s'éloigne de la bordure de recouvrement, la craje devient plus compacte et moins perméable et on peut estimer que la nappe est ainsi limitée par une barrière imperméable à peu de distance du recouvrement.

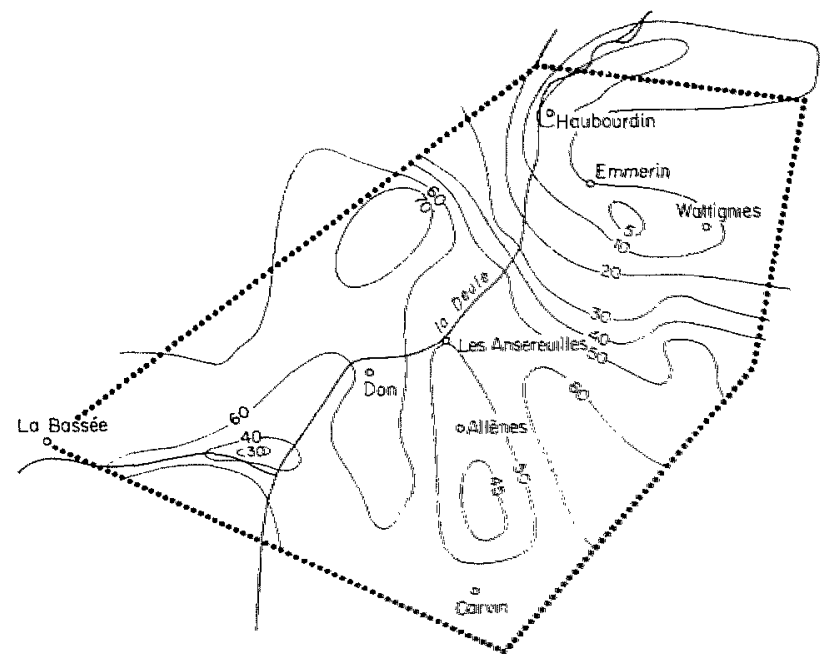

Firt.

Isopaques de la craie.

\section{Résolution analogique du problème.}

Nous avons montré comment le simulateur pouvait à la fois représenter les parties libres et captives de la nappe aquifère. Il convient maintenant d'insister surtout sur les restrictions apportées à la représentation proprement dite de la nappe. Du fait de la grande extension du bassin d'alimentation, il n'était pas possible de le représenter, ni possible de simuler correctement les précipitations atmosphériques. Aussi, grâce à l'application du principe de superposition, s'est-on limité à la zone la plus intéressante. Cette zone est définie au nord-ouest et ou sud-est par les barrières imperméables, sensiblement Jes lignes La Bassée-Santes et Carvin-Seclin, au nord-est par le seuil du Mélantois et au sudouest par la ligne La Bassée-Carvin; l'équation de diffusivité étant linéaire en $\mathrm{H}$, la solution du problème général s'obtient alors en additionnant la solution de l'état primitif et celle que nous donne la perturbation considérée isolément dans la zone restreinte, pourvu que les deux solutions particulières correspondent à des conditions aux limites dont la résultante est identique à cclles du problème général. Ainsi devons-nous vérifier que la solution correspondant à la perturbation étudiée seule est pratiquement nulle sur les limites de la zone étudiée. En fait, les conditions d'application du problème sont plus souples : compte tenu de la circulation générale des eaux, on peut admettre une baisse du niveau à la limite sud d'alimentation, qui aurait pour effet d'augmenter l'entrée d'eau dans la zone à laquelle nous nous intéressons. Au seuil du Mélantois, un abaissement de niveau diminuant le débit de fuite vers Lille est par contre difficilement acceptable, tandis qu'une remontée du niveau $y$ provoquerait une augmentation du débit de fuite, done une diminution du niveau, dans la zone étudiée, au plus égale à la remontée constatée au seuil du Mélantois.

La zone étudiée a été découpée en mailles régulières de $1500 \mathrm{~m}$ de côté; l'aquifère étant hétérogène, il a été nécessaire d'attribuer à chaque maille une transmissivité moyenne à partir de la carte d'isotransmissivités; le coefficient d'emmagasinement a été pris constant et égal à $5.10^{-4}$ dans les parties captives; dans les parties libres, il est égal à la porosité efficace, laquelle a été un paramètre pour chaque expérience, oscillant entre 1 et $4 \%$. Le maillage ainsi utilisé est assez grossier et ne fournit que les pressions ou les cotes moyennes dans une maille. Tous les puits se trouvant dans le secteur correspondant à une maille sont groupés sur l'analyseur en une seule fonction production ou une seule fonction injection. C'est le grand rapport entre les

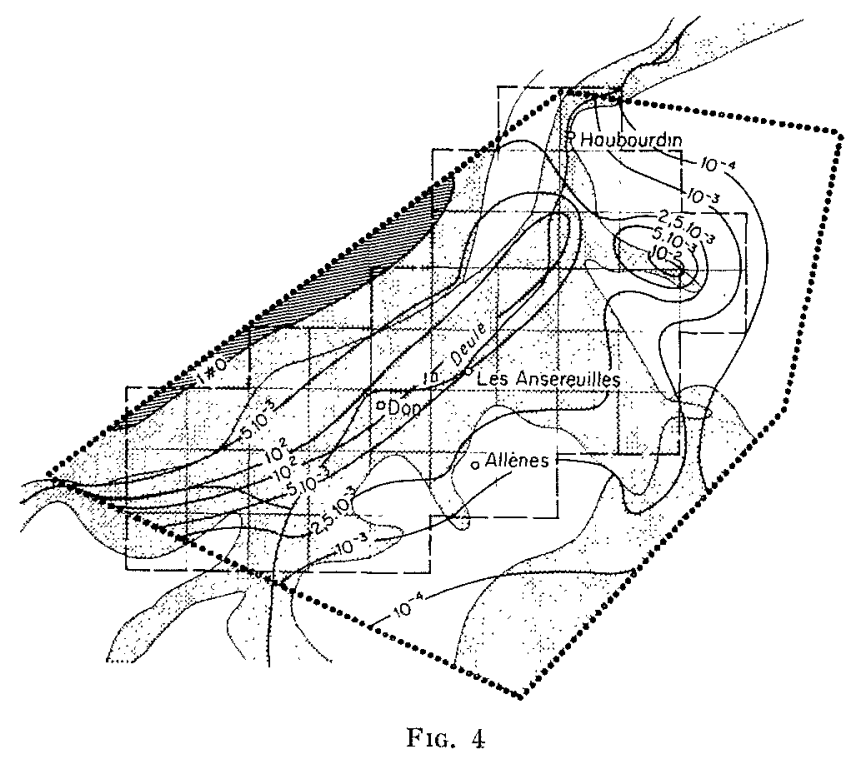

Carte d'isotransmissivité. $\mathrm{T}=(h k / \mu) \mathrm{Q} g$. 


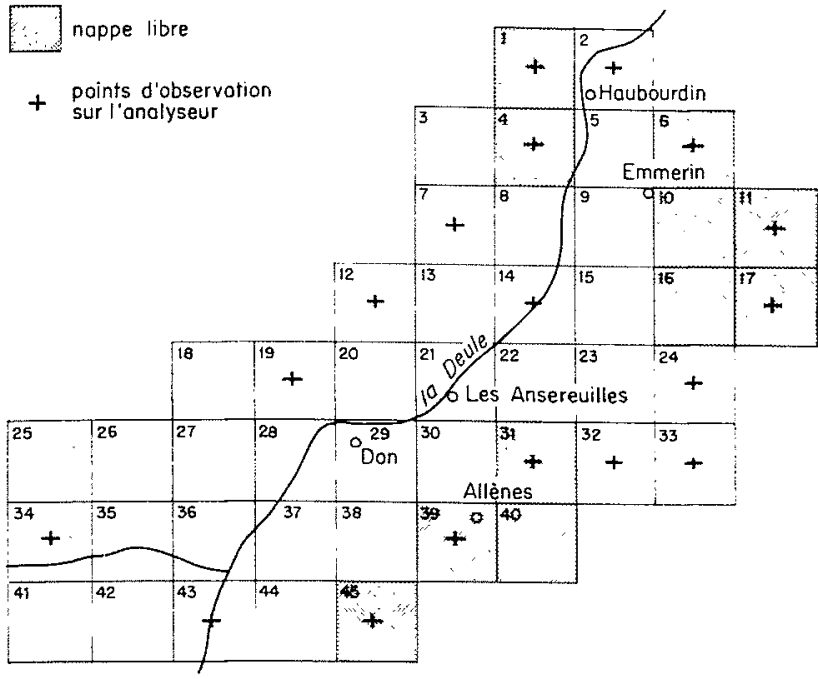

FIG. 5

Maillage analyseur.

réserves des parties libres et des parties captives (environ 1000) qui nous a obligé à prendre un maillage aussi lâche, compte tenu de la plus petite capacité qu'il était possible de représenter.

Etant donné le caractère cyclique des opérations de production et d'injection, nous aurions pu n'étudier la perturbation considérée que durant une année. Afin, de mieux observer la stabilisation ou la non stabilisation du phénomène il a paru préférable de considérer deux cycles et quelquefois plus.

\section{Enoncé des solutions envisagées.}

Etant donné les impératifs concernant la mise en cuvre de la réalimentation de la nappe, le lieu d'injection a été primitivement fixé dans la région de Don; pour des raisons industrielles, l'éventail des solutions envisagées a été limité au départ à deux zones possibles pour le soutirage, la région des Ansereuilles (où la nappe est captive) et la région d'Allènes-les-Marais (oì la nappe est libre). Les emplacements géographiques ainsi précisés, la ventilation et l'importance des débits annuels ont donné lieu à deux cas très différents :

a) soutirage durant toute l'année à raison de $40000 \mathrm{~m}^{3} / \mathrm{j}$

b) soutirage durant les quatre mois de la saison sèche à raison de $40000 \mathrm{~m}^{3} / \mathrm{j}$.

Dans les deux cas, l'injection ne se faisait que durant les huit mois humides, avec un débit cumulé égal dans les deux cas au soutirage annuel. La combinaison des emplacements de soutirage et de la répartition des débits dans le temps a ainsi donné lieu à six hypothèses :

$1^{\circ}$ injection à Don de $60000 \mathrm{~m}^{3} / \mathrm{j}$ pendant huit mois, prélèvement aux Anserenilles, de $40000 \mathrm{~m}^{3} / \mathrm{j}$ pendant toute l'année;

$2^{\circ}$ injection à Don de $60000 \mathrm{~m}^{3} / \mathrm{j}$ pendant huit mois, prélìvement à Allènes-les-Marais de $40000 \mathrm{~m}^{3} / \mathrm{j}$ pendant toute l'année;

$3^{\circ}$ injection à Don de $60000 \mathrm{~m}^{3} / \mathrm{j}$ pendant huit mois, prélèvement de $40000 \mathrm{~m}^{3} / \mathrm{j}$ pendant toute l'année, répartis également entre les Ansereuilles et Allènes;

$4^{\circ}$ injection à Don de $20000 \mathrm{~m}^{3} / \mathrm{j}$ pendant huit mois, prélèvement de $40000 \mathrm{~m}^{3} / \mathrm{j}$ aux Ansereuilles pendant les quatre autres mois;

$5^{\circ}$ injection à Don de $20000 \mathrm{~m}^{3} / \mathrm{j}$ pendant huit mois, prélèvement de $40000 \mathrm{~m}^{3} / \mathrm{j}$ à Allènes pendant les quatre autres mois;

$6^{\circ}$ injection à Don de $20000 \mathrm{~m}^{3} / \mathrm{j}$ pendant huit mois, prélèvement de $40000 \mathrm{~m}^{3} / \mathrm{j}$ pendant les quatre autres mois, répartis également entre les Ansereuilles et Allènes-lesMarais.

D'autres cas, ne répondant pas aux préoccupations premières du Service des Mines ont été étudiés. Nous ne les présentons pas, faute de place et de temps.

\section{Discussion des résultats.}

Il ressort des expériences 1,2 et 3 que l'implantation de puits de soutirage dans la région à très bonne transmissivité des Ansereuilles n'est pas avantageuse; en effet, cette implantation masque l'effet que l'injection pourrait avoir au point critique du seuil du Mélantois, près d'Haubourdin; de plus, l'injection a une tendance très nette à provoquer un relèvement général des niveaux dans le sud-ouest de la vallée, à la limile du bassin d'alimentation dans la région de La Bassée.

\section{Variations extrêmes des niveaux}

\begin{tabular}{|c|c|c|c|c|}
\hline \multirow{5}{*}{$\begin{array}{l}\text { Exp. 1 . } \\
\text { Exp. } 2 \ldots \\
\text { Exp. } 3 \ldots\end{array}$} & \multicolumn{2}{|c|}{$\begin{array}{l}\text { Haunoundin } \\
\text { (Hauteur de) } \\
\text { la eraie } 5 \mathrm{~m} \text { ) }\end{array}$} & \multicolumn{2}{|c|}{$\begin{array}{c}\text { La Bassíb } \\
\text { (Hauteur de) } \\
\text { la craie } 60 \mathrm{~m} \text { ) }\end{array}$} \\
\hline & & en $\mathrm{m}$ & $\begin{array}{l}\text { en } m \\
-3\end{array}$ & $\begin{array}{l}\text { en } \mathrm{m} \\
+12\end{array}$ \\
\hline & 0 & $-3,60$ & -3 & +12 \\
\hline & $-0,40$ & $+0,80$ & -1 & +20 \\
\hline & 0 & $-1,40$ & $-2,20$ & +16 \\
\hline
\end{tabular}


EXPÊRIENCE 1. - Échelle des temps : 1 carreau $=2,4$ mois

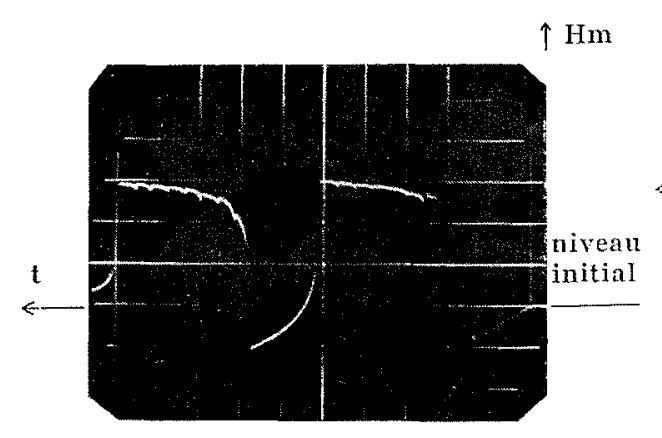

Don (maille 29)

1 carreau $=10$ mètres

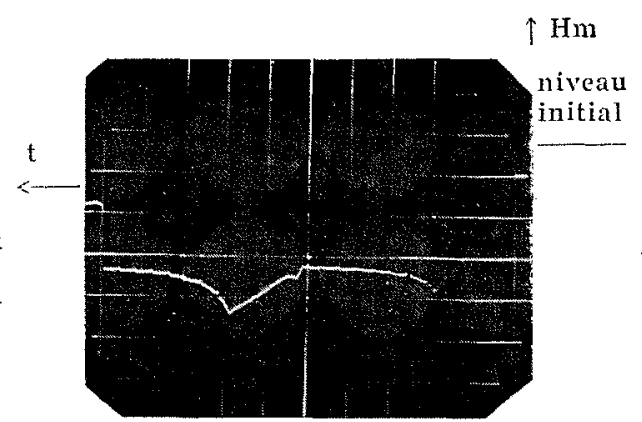

Les Ansereulles (maille 21)

1 carreau $=10$ mètres

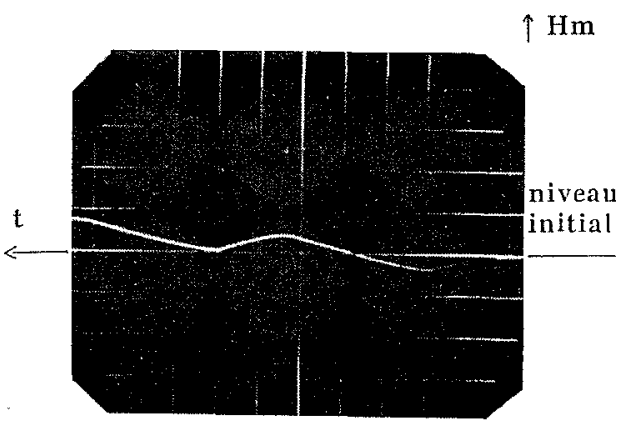

Altènes (maille 39)

1 carreau $=2$ mètres

\section{EXPÉRIENCE 2. - Échelle des temps : 1 carreau $=2,4$ mois}

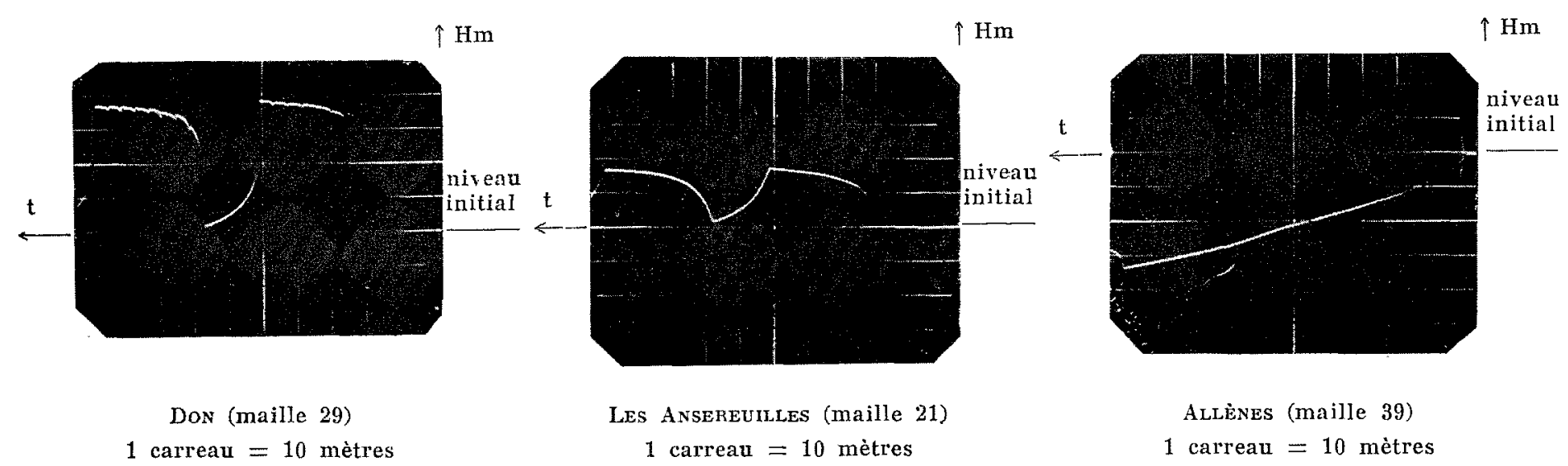

EXPÉRIENCE 3. - Échelle des temps : 1 carreau $=2,4$ mois

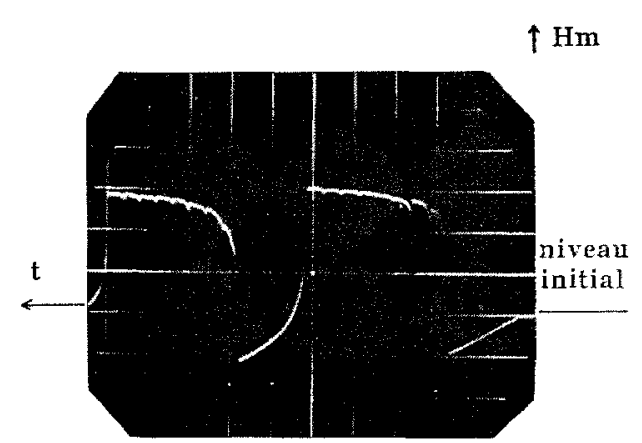

Don (maille 29)

1 carreau $=10$ mètres

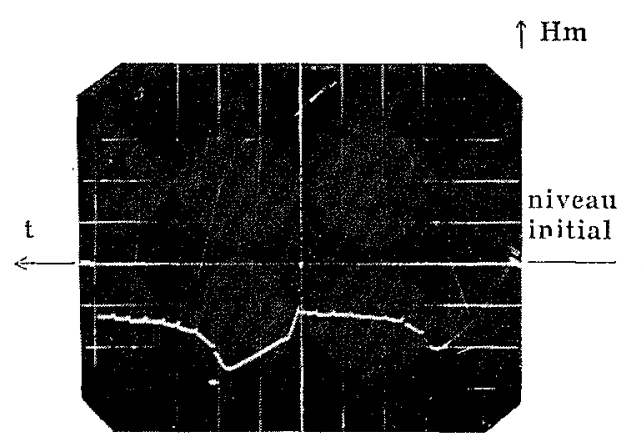

Les Ansereulles (maille 21) 1 carreau $=10$ mètres

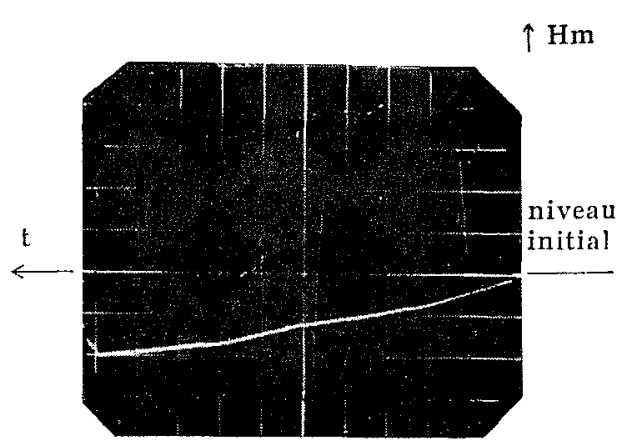

Allènes (maille 39)

1 carreau $=10$ mètres 
EXPÉRIENCE 4. - Échelle des temps : 1 carreau $=2,4$ mois

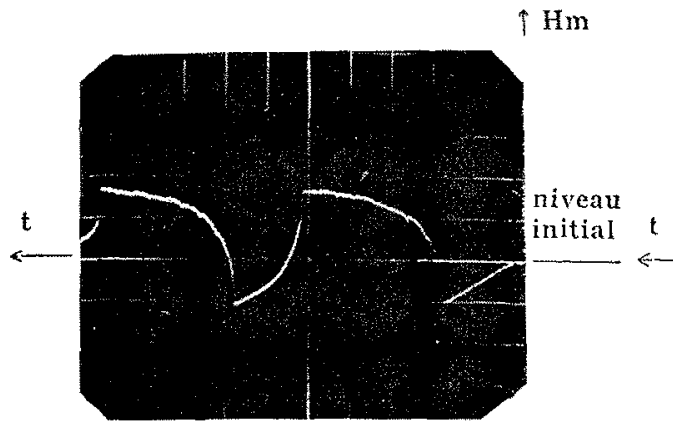

DoN (maille 29)

1 carreau $=10$ mètres

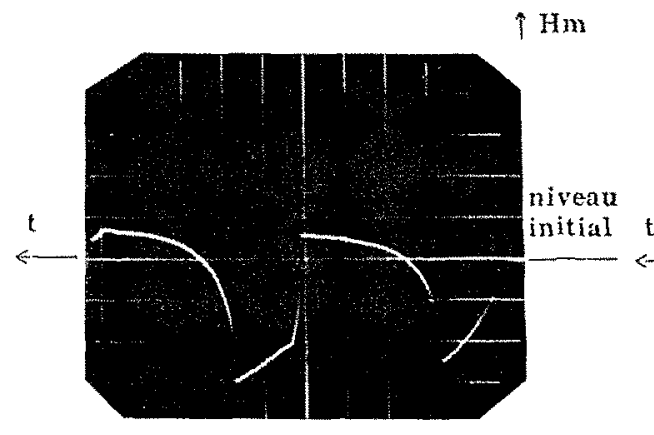

Les Ansereurles (maille 21) 1 carreau $=10$ mètres

$1 \mathrm{Hm}$

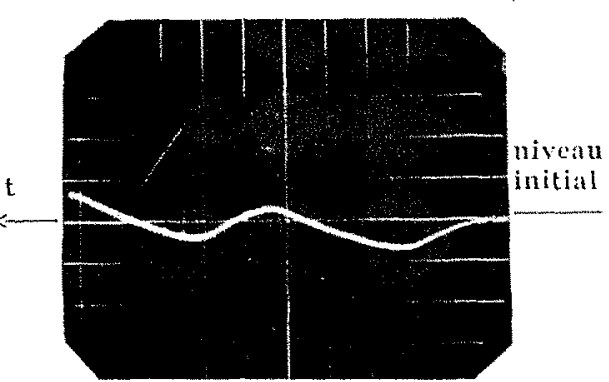

Alzènes (maille 39)

1 carreau $=2$ metres

EXPÉRIENCE 5. - Échelle des temps : 1 carreau $=2,4$ mois

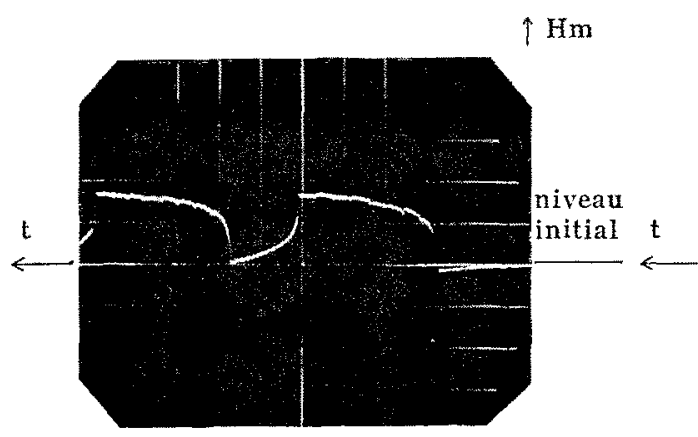

DoN (maille 29)

1 carreau $=10$ mètres

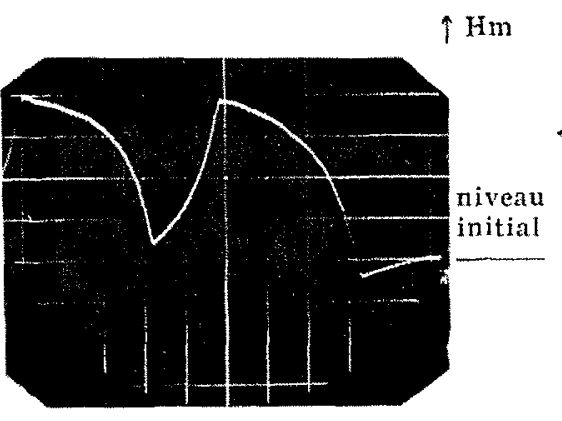

Les Anseneurlzes (maille 21)

1 carrean $=2$ mètres

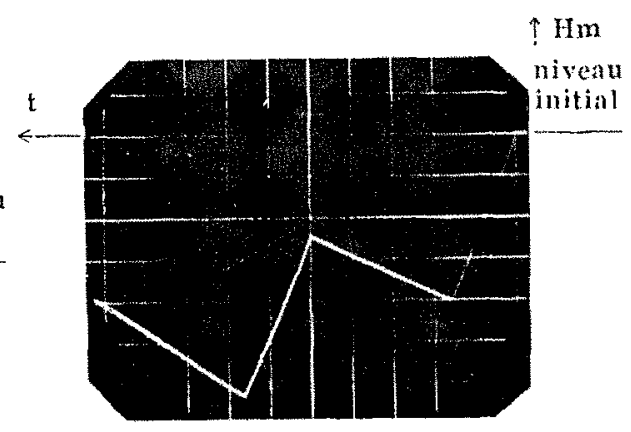

ALtìnes (maille 39)

1 carreau $=4$ mètres

EXPÉRIENCE 6. - Échelle des temps : 1 carreau $=2,4$ mois

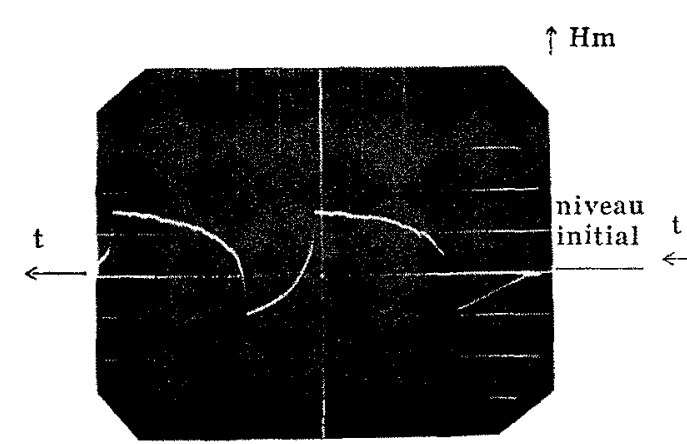

Don (maille 29)

1 carreau $=10$ metres

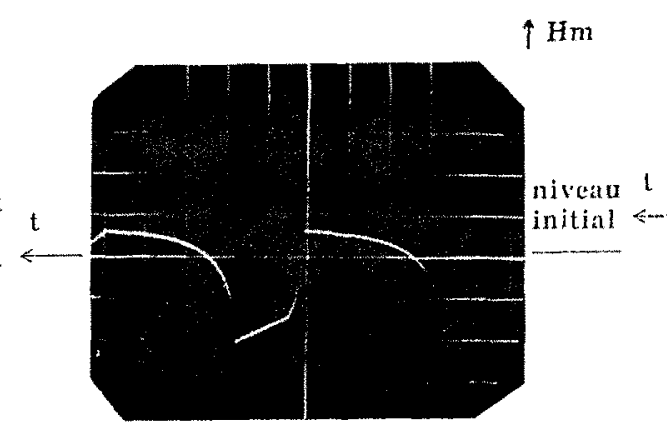

Les ANsereuilees (maille 21) 1 carreau $=10$ mètres $\uparrow \mathrm{Hm}$

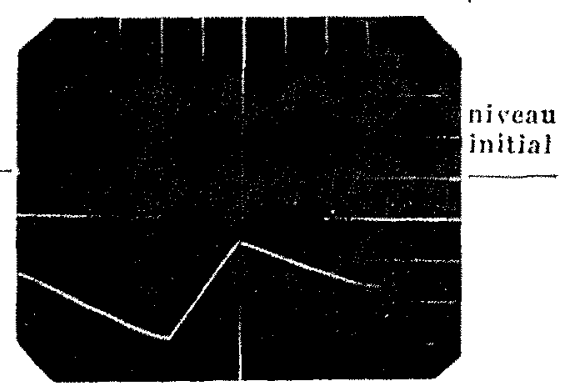

Athises (maille 39)

1 carreau $=2$ métres 
Dans les expériences 4,5 et 6 , où le soutirage n'a lieu que durant les quatre mois d'été, avec par suite une production cumulée réduite des $2 / 3$ par rapport aux expériences 1,2 et 3 , les variations de niveau sont évidemment moins importantes. Mais, cette fois-ci, du fait que injection et soutirage sont totalement alternés, l'injection a un effet notable sur le niveau du seuil du Mélantois, niveau qui oscille périodiquement avec un décalage de temps (qu'il est possible de mesurer) caractéristique de la transmissivité.

\section{Variations extrêmes des niveaux}

\begin{tabular}{|l|cc|cc|}
\hline & $\begin{array}{c}\text { Haubourdin } \\
\text { (Hauteur de) } \\
\text { la craie 5 m) }\end{array}$ & $\begin{array}{c}\text { La Basske } \\
\text { (Hauteur de) } \\
\text { la craie } 60 \mathrm{~m} \text { ) }\end{array}$ \\
\hline en $\mathrm{m}$ & en $\mathrm{m}$ & en $\mathrm{m}$ & en $\mathrm{m}$ \\
Exp. 4... & $-0,40$ & -1 & $-3,20$ & $+5,60$ \\
Exp. 5... & $-0,20$ & $+0,20$ & $-0,80$ & $+6,50$ \\
Exp. 6... & $-0,60$ & $-0,20$ & $-2,40$ & +5 \\
\hline
\end{tabular}

En considérant à la fois le niveau au seuil du Mélantois et les variations de ce niveau à la limite du sud-ouest, il apparaît que, la question d'implantation des puits mise à part, le cas de l'alternance totale des débits est préférable.

\section{III. - CONCLUSIONS}

\section{Conclusions particulières à l'étude.}

Les réponses fournies par le simulateur aux hypothèses envisagées en premier pour résoudre le problème d'un soutirage supplémentaire ne sont pas toutes acceptables; et même les meilleures (cxpériences 5 et 6 ) ne répondent qu'imparfaitement aux conditions exigées. A ce stade cependant, le simulateur a déjà permis d'effectuer le classement des solutions envisagées, de préciser les ordres de grandeur, de mettre en évidence les effets des variations de la transmissivité, en résumé de cerner le problème. Ainsi, dans un second stade, compte tenu des premiers résultats, il nous a été possible de proposer des solutions améliorées quant à l'emplacement des puits. Cela ne répond pas aux premiers désirs du Service des Mines, mais montre comment le simulateur peut être utilisé quand il s'agit d'optimiser. Il est évident néanmoins que des informations supplémentaires, à savoir de nouveaux forages et de nouveaux tests de transmissivité, sont nécessaires si l'on veut aborder le problème de façon plus fine sur le simulateur. Un nouveau maillage serait alors à étudier. Cette étude est ainsi un bon exemple de la souplesse d'emploi du simulateur lorsque les informations sont suffisamment rares pour qu'une étude numérique soit vraiment efficace.

\section{Conclusions générales sur l'utilisation $d u$ simulateur.}

Les simulateurs R. C. ont été primitivement concus pour l'étude de la diffusion de la chaleur, puis appliqués à l'étude des gisements d'huile sous-saturée et de leurs aquifères adjacents. La part prise par ces aquifères dans l'étude des gisements sur analyseur électrique a souvent été prépondérante, le gisement d'huile n'étant plus considéré que comme le lieu d'application d'une condition de débits. L'analyseur électrique a ainsi permis d'étudier des aquifères communs à plusieurs gisements d'huile. L'application que nous en avons faite dans le cas de la nappe aquifère de la craie de la vallée de la Haute-Deule est l'illustration d'un nouveau champ d'utilisation de l'appareil.

La simulation des nappes phréatiques présente certains caractères particuliers, tels la linéarisation des équations de l'écoulement et la restriction relative au rapport maximal des réserves entre parties libres et captives; néanmoins, malgré ces approximations et malgré le nombre limité de mailles et de puits, l'étude des mouvements transitoires peut être faite dans des cas très complexes d'hétérogénéités, avec failles et limites imperméables de forme quelconque et avec limites à potentiel constant. Ces études peuvent avoir un caractère d'application ou un caractère théorique. En fait, les problèmes de diffusivité, que ce soit dans les gisements d'huile ou dans les nappes aquifères, peuvent se poser de différentes façons.

Si l'on considère connues les caractéristiques de la nappe et les conditions de débits exigées, le problème est alors le problème du choix des emplacements de puits et de la répartition des débits, compte tenu de certains impératifs, ou tout problème plus simple de même type.

$\mathrm{Si}$ l'on dispose de puits d'observation et de mesures d'interférences ou de remontées de pression, le problème est alors la détermination des caractéristiques de la nappe, surtout des transmissivités, en vue d'une utilisation ultérieure pour un problème de prévisions.

L'étude rationnelle et méthodique des problèmes de diffusivité dans les nappes aquifères peut 
ainsi permettre de résoudre, grâce au simulateur, le problème de l'économie des aqueducs, en déterminant de façon précise, en fonction des caractéristiques, quel est le déphasage de temps nécessaire entre le début de l'injection au point de départ $\mathrm{A}$ et le commencement du soutirage au point d'arrivée souhaité $B$.

En conclusion, la souplesse du simulateur est assez grande pour que l'on puisse simplement, en même temps :

$1^{\circ}$ utiliser au mieux des renseignements incomplets ou imprécis pour lesquels la précision d'un calculateur numérique est illusoire;

$2^{\circ}$ mener à terme une étude d'ensemble sommaire dans un temps très rapide sans engager de frais importants;

$3^{\circ}$ réaliser l'étude précise de l'optimisation de certains paramètres.

\section{Bibliographie}

Paschisis (V.) and Baker (H. D.). - A method for determining unsteadystate heat transfer by means of electrical analogy. Trans. Am. Soc. Mech. Engrs., Vol. 64, p. 105-112, 1942.

Patrenson (O. L.) et autres. - High speed electronic reservoir analyzer. Drilling and Production practice, p. $47-63,1951$.

WatenLot (G.). - Observations sur les mappes aquileres de la craie du Nord de la France. Association Internationale des Hydrogéologues. Congrès de Liège 1958. Mém., tome 2, Paris. B.R.G.M., p. 129-134.

Karplus (W. J.) and Sonoka (W. W.). - Analog methods. Chapitre 10: finite difference networks. p. 323-383. MacGraw Hill Book Company Ine., New York, 1959.

Krylov (A.P.) et autres. - Projet d'exploitation des gisements de pétrole. Chapitre 22 : Modèles électriques. Gostoptekizdat, Moscou, 1962.

Scholler (H.). - Les eaux souterraines. Masson et Cie, 1962.

\section{I S C US S I O N}

Président: M. Chapolthien

M. le Président remercie M. Lagarde de sa très intéressante communication et des renseignements pratiques qu'il en a déduits.

Il a un doute sur la fidélité de l'appareil dont parle M. LAGARDE, car le milieu poreux est un volume hétérogène et le simulateur est une longueur homogène. De plus, M. Lagarde a employé l'équation de Depuit, qui est la première équation de l'hydlaulique souterraine, mais qui a été abandonnee depuis un certain temps par les hydrauliciens. En effet, cette équation suppose que les pentes sont très faibles et les courants permanents, alors qu'on tend maintenant à considérer que les nappes ont des mouvements doucement variables, mais non des mouvements permanents avec une pente pratiquement négligeable, ce qui aboutirait à des absurdités : l'hypothèse de Dupuit arrive notamment à la conclusion que si l'on ouvre un trou dans la nappe, on se raccorde par $O$ et le débit devient infini.

Les tarages que M. Lagarde a pu effectuer et qui consistaient à confronter la similitude avec la réalité donnent-ils le moyen de rejeter ces appréhensions et de croire à une fidélité suffisante du simulatenr par rapport au terrain?

M. LAGAibe indique que la confrontation entre la similitude et la réalité n'a pu être faite dans le cas de la nappe aquifère dont il vient de parler, car cette étude constitue un projet qui n'a pas encore vu le jour, mais des confrontations $\dot{a}$ court ferme concernant, des gisements de pétrole ont déjà prouvé l'efficacité du simulateur, convenablement u'ilisé. Cette dernière remarque amène à répondre au doute émis par $M$. le Président sur la validité de l'équation de Dupuit. Il se trouve que, si les hypothèses qui ont présidé à son établissement sont approchèes, la formule elle-mème est exacte et une démonstration rigoureuse figure dans Polubarinova-Kochina. Pour notre part, nous avons seulement supposé que la pente de la nappe libre était faible. Etant donné que, dans la partic libre comme dans la pattie captive, le simulateur ne nous donne gue des variations moyennes sur une surface importante $(1,500 \times 1,500 \mathrm{~km})$, il est évidemment hors de question de vouloir simuler le comportement exact anx puits mêmes. Il est, par contre, important que le comportement moyen résultant donne, aux endroits critiques tel que le seuil du Mélantois, des résnltats très proches de la réalité, ce qui est le cas et peut-être démontré. Il est évident que dans le cas où les pentes du mur de l'aquifère et de la surface libre sont trés importantes, le simulateur ne prenant pas en compte les efrets de la gravité, ses résultats peuvent être fort erronés.

M. Ruby, en réponse à ce que vient de dire M. le Président Chapouthien, voudrait faire remarquer que si les équations de Dupuit sont moins utilisées en hydrauliques souterraine, les hypothèses de Dupuit sont conservèes (écoulement à faible gradient - surfaces équipotentielles assimilables à des cylindres à génératrices verticales - faibles perturbations des conditions aux limites par rapport aux conditions initiales) môme dans les équations mises au point pap Theiss qui introduisent la variable «temps».

Si done on étudie des phénomènes où les perturbations des conditions aux limites sont faibles par ripport aux conditions initiales, l'analogie présentée est valable, pourvu qu'on ne considere les effets d'unc perturbation cu'à une certaine distance de celle-ci.

Le modéle analogique R.C. nécessite-t-il que l'on connaisse a priori les caractéristiques hydrodynamiques des lerrains (transmissivité - capacité d'écoulement), auguel cas il faut ajouter, au prix de l'etude. Ia determination de ces caractéristicues par des tests préeis (essais de pompages -..- essais Lefrane dans des sondages, etc.) et coúteux, ou bien peut-on reproduire sur le modèle et par tâtonnoment, un état initial donné de nappe, déconlant de mesures faites dans des puits ou 
piézomètres lors de l'étude générale de celle-ci? (Cet état initial étant d'ailleurs un état en régime non permanent, dans les conditions naturelles habituelles.)

D'autre part, il parait souhaitable à $M$. RuBy de ne pas appeler $k$ le facteur $\mathrm{T} / \mathrm{S}$ (transmissivité divisée par capacité d'écoulement), ce symbole $k$ étant habituellement utilisé en hydraulique souterraine pour représenter la perméabilité à l'eau d'un terrain (coefficient de Darcy).

M. LAGarde répond qu'effectivement l'utilisation du simulateur ne nécessite pas la connaissance des caractéristiques hydrodynamiques des terrains, mais il est bien évident qu'une telle connaissanee, mème partielle, facilite énormément le travail qu'il faut fairc sur le simulateur pour recréer des conditions données aux puits. Ce cas intermédiaire, où les connaissances ne sont que partielles, est le cas général d'utilisation du simulateur, et si, dans l'étude qui a été décrite, on a admis une configuration donné de la carte des isotransmissivités, la seule raison en est dans le caractère d'avant-projet et de dégrossissage de cette étude. Cette carte a néanmoins été établie à partir de tous les tests et de toutes les informations géologiques disponibles seule question qui se pose est de savoir si leur nombre etait suffisant; M. LACARDE pense que des informations supplémentaires ne modifieraient pas beaucoup Ia tendance indiquée par la carte. En fait, le problème général de la détermination des caractéristiques d'une couche connaissant certaines mesures aux seuls puits de la couche est une des applications principales du simulateur en vue de létablissement des prévisions. Ce problème peut ètre abordè par voíe mathématique. M. Jacquard, à l'I.F.P. a déf cas de l'interprétation des tests de Theiss en géométrie circulaire. Pour finir de repondre à M. Runy, M. Lagarde est d'accord avec lui pour dire que la désignation de Ja diffusivité hydraulique rapport $T / S$ est mauvaise et ne s'est jusqu'ici justiffée en terminologie pétrolière que par l'ignorance rolontaite du coeffeient de Darcy au profit du coefficient de perméabilité $k$.

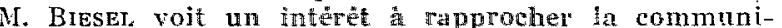
cation de M. Lagarde de leâtude faite il $y$ a quelques années sur le problème tout à faid similaire de la nappe du chott Chergai. Cette étude avait été exécutée par M. Zaoui sur machine électronique numérique et il avait été nécessaire d'introduire l'influence de la per- méabilité très faible du toit argileux de la nappe. Les débits passant à travers ce toit n'ètaient pas négligeables, étant donné les surfaces en jeu et, bien entendu, ils variaient avec les pressions hydrostatiques dans la nappe.

Lá difficulté était que les variations de ce débit sont une fonction complexe de l'histoire des variations de pressions et pas seulement de leurs valeurs instantanées. En effet, les dépressions (ou compressions) se propagent relativement lentement, suivant la verticale dans l'épaisseur du toit (en suivant d'ailleurs. nne équation du type de celle de la chaleur). Par exemple, au début d'une dépression qui se stabilise ensuite, le débit fourni par le toit est beacoup plus grand que par la suite.

M. LaGande pourrait-il nous dire si de tels types de lois pourraient être introduits dans son modèle analogique?

M. Biesel demande d'autre part, le prix de revient d'un tel appareil et d'nne telle éturle.

M. Lagarne dit que l'étude de phénomènes tels que coux évoqués par M. Biesel est certainement possible, puisqu'ils mettent en jeu la diffusivité des terrains. En fait, tout dépend de la finesse du maillage et du nombre de points où l'on veut imposer certaines conditions de débit, le simulateur étant limité en mailles et puits.

Quant au prix de l'appareil, il n'est pas possible de le donner; il est surtout fonction du prix des relais magnétiques intervenant dans le système de télécommande.

M. Bieser indique que, dans le phénomène $\mathrm{du}$ choc thelmique, si on change brusquement la température sur un mur en liaison avec une autre température de l'autre còté, il $y$ a un certain transitoire assez long avant que le gradient de température deviemne normal à travers le mur. S'il $y$ a une dépression, le gradient devient normal plus tôt que ce qui seraít donné par la formule de Darcy appliquée à l'épaisseur du toit. Puis, petit à petit, asymptotiquement, si la pression est redevenue constante, le débit apporté par le toit redevient celui donué par la formule de Darcy.

M. Laciande indique que le choc thermique peut être représenté sur l'analyseur électrique en chargeant les capacités à deux tensions différentes. L'appareil reproduit alors le transitoire qui a lieu quand on met les deux côtés du mur en liaison.

M. le Président remercie à nouveau M. Lagarde pour sa brillante communication.

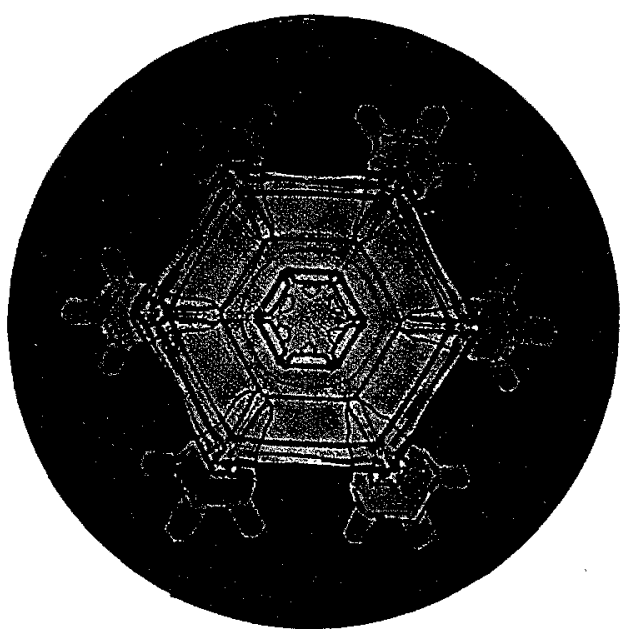

